\title{
Resultados de la ventilación asistida ajustada neuronalmente no invasiva y la presión positiva continua nasal en recién nacidos prematuros: revisión sistemática y metanálisis Outcomes of noninvasive neurally adjusted ventilatory assist and nasal continuous positive airway pressure in preterm infants: a systematic review and meta-analysis
}

\author{
Yaya Xu $u^{a}$ (D) Xiaodong Zhu ${ }^{a}$ (D) Xiangmei Kong ${ }^{a}$ (D) , Jiru $L i^{a}(\mathbb{D})$
}

\section{RESUMEN}

Introducción: los beneficios de la ventilación asistida ajustada neuronalmente (NAVA) en los recién nacidos prematuros son inciertos. El objetivo de este estudio fue explorar si la NAVA no invasiva (NIV) era más beneficiosa para los recién nacidos prematurosquela presión positiva continua nasal (NCPAP).

Diseño del estudio: metanálisis de tres ensayos clínicos: dos ensayos controlados aleatorizados y un estudio de grupos cruzados. Se comparó la NAVA-NIV con la NCPAP y se informó sobre el fracaso del tratamiento, la mortalidad y los eventos adversos como resultados principales. Resultados: tres estudios con 173 pacientes (89 recibieron NAVA-NIV) cumplieron los criterios deinclusión en estemetanálisis. Noseobservaron diferencias en el fracaso del tratamiento entre la NAVA-NIV y la NCPAP (razón de riesgos [RR] $=1,09$; intervalo de confianza [IC] del $95 \%=0,65-1,84$; diferencia de riesgos $=0,02$; IC95\% $\left.=-0,10-0,14 ; I^{2}=33 \% ; P=0,23\right)$. De manera similar, no hubo diferencias en la mortalidad $(\mathrm{RR}=1,52$; IC95\% = 0,51-4,52; no aplica heterogeneidad). En comparación con la NCPAP, la NAVA-NIV redujo significativamente el uso de cafeína $(R R=0,85$; IC 95\% = 0,74-0,98; $\left.\mathrm{I}^{2}=71 \% ; P=0,03\right)$.

Conclusiones: en comparación con la NCPAP, no hay evidencia suficiente para sacar una conclusión sobre los beneficios o daños de la NAVA-NIV en los recién nacidos prematuros. Los hallazgos de esta revisión deben confirmarse en ensayos clínicos con una metodología rigurosa y potencia adecuada.

Palabras clave: ventilación no invasiva, recién nacido prematuro, respiración artificial, respirador interactivo, displasia broncopulmonar.

http: / / dx.doi.org/10.5546/ aap.2022.89

Texto completo en inglés:

http: / / dx.doi.org/10.5546/ aap.2022.eng.89

Financiamiento:

Ninguno.

Conflicto de intereses: Ninguno que declarar.

Recibido: 7-1-2021

Aceptado: 11-7-2021
Cómo citar: Xu Y, Zhu X, Kong X, Li J. Resultados de la ventilación asistida ajustada neuronalmentenoinvasiva y la presión positiva continua nasal en recién nacidos prematuros: revisión sistemática y metanálisis. Arch Argent Pediatr 2022;120(2):89-98.

\section{Siglas y abreviaturas}

AEdi: señal de actividad eléctrica del diafragma.

DBP: displasia broncopulmonar.

DM: diferencia media.

DR: diferencia de riesgos.

ECA: ensayos controlados

aleatorizados.

IC: intervalo de confianza.

NAVA-NIV: ventilación asistida ajustada neuronalmente (neurally adjusted ventilatory assist) no invasiva. NCPAP: presión positiva continua nasal (nasal continuous positive airway pressure)

OR: razón de posibilidades.

PPFE: presión positiva al final de la espiración.

PS: presión de soporte.

RR: razón de riesgos.

SDRN: síndrome de dificultad respiratoria neonatal.

UCI: unidad de cuidados intensivos. VMI: ventilación mecánica invasiva.

\section{INTRODUCCIÓN}

Según los informes, el parto prematuro es la principal causa (en aumento) de muerte en los niños en todo el mundo; actualmente, resulta en 1000000 de muertes anuales, y el síndrome de dificultad respiratoria neonatal (SDRN) es la causa más frecuente de muerte prematura. ${ }^{1-3} \mathrm{En}$ los últimos años, los médicos priorizaron la aplicación temprana de la ventilación no invasiva; ahora, la presión positiva continua nasal (NCPAP) es uno de los métodos más frecuentes para 
tratar el SDRN. ${ }^{4}$ Sin embargo, algunos estudios demostraron que la incidencia de neumotórax en los pacientes que recibieron NCPAP era más elevada que en aquellos que recibieron ventilación mecánica no invasiva y ventilación mecánica invasiva (VMI). ${ }^{5}$

La ventilación asistida ajustada neuronalmente (neurally adjusted ventilatory assist, NAVA) se usa para monitorear la actividad eléctrica del diafragma (AEdi), captar las necesidades de ventilación reales del paciente y brindar cierto soporte ventilatorio en el acto según la intensidad de la señal de la AEdi. En teoría, el inicio de la NAVA y la conversión de inhalación y exhalación están impulsados directamente por la electromiografía del diafragma, que maximiza la sincronización entre el paciente y el equipo. ${ }^{6}$ Los recién nacidos, en especial los prematuros, podrían tener inmadurez del centro respiratorio, y los mecanismos de respuesta respiratoria podrían estar afectados por enfermedades respiratorias, las que pueden perturbar la AEdi. ${ }^{7}$

Pocos estudios han comparado el pronóstico de la NAVA no invasiva (NAVA-NIV) y la NCPAP. Sin embargo, nunca se hizo una revisión sistemática y metanálisis centrada en los recién nacidos prematuros. Nuestra revisión sistemática y metanálisis se enfocó en los recién nacidos prematuros que requieren NAVA-NIV o CPAP y las complicaciones asociadas con la ventilación no invasiva.

El objetivo de este estudio fue llevar a cabo una revisión sistemática de los artículos sobre NAVANIV y CPAP para investigar las diferencias en los resultados clínicamente relevantes en los recién nacidos prematuros con distintos patrones respiratorios.

\section{MÉTODOS}

Obtuvimos datos, de manera sistemática, sobre los resultados de los recién nacidos prematuros que recibieron NAVA-NIV o CPAP de PubMed (desde 1941 hasta el 4-12-2020), Embase (desde 1947 hasta el 4-12-2020), Web of Science (desde 1960 hasta el 4-12-2020), la biblioteca Cochrane (edición 12 del 12-12-2020) en forma de estudios informados.

Los recién nacidos prematuros se definieron como bebés nacidos con una edad gestacional de menos de 37 semanas, según los criterios desarrollados por el Taller del Instituto Nacional de Salud del Niño y Desarrollo Humano en 2005. ${ }^{8}$

Se buscaron los siguientes términos $\mathrm{MeSH}$ y texto libre: ventilación asistida ajustada neuronalmente*[título/resumen] O ventilación asistida proporcional*[título/resumen] O soporte ventilatorio interactivo*[título/resumen] O soporte ventilatorio*[título/resumen] y presión positiva continua nasal. Además, se aplicaron limitadores específicos de la base de datos para ensayos controlados aleatorizados (ECA) y recién nacidos. No se aplicaron restricciones de idioma. También se hizo una búsqueda en las listas de referencias de los artículos seleccionados para su inclusión en esta revisión para identificar artículos adicionales relevantes. También se hizo una búsqueda en los resúmenes de conferencias para encontrar algún estudio relevante no publicado. Todas las búsquedas se evaluaron según la declaración PRISMA sobre los ítems de referencia para la publicación de revisiones sistemáticas y metanálisis. Debido a la naturaleza de este estudio, no se requirió la aprobación de un comité de ética.

\section{Criterios de inclusión y exclusión}

Se incluyeron los estudios que cumplieron con los siguientes criterios: (a) ECA o ensayo de grupos cruzados que comparara la NAVA-NIV y la NCPAP en recién nacidos prematuros; (b) recién nacidos prematuros con SDRN aleatorizados para recibir soporte respiratorio con CPAP frente a NAVA; y notificación de más de uno de los siguientes parámetros de resultados principales: requerimiento de oxígeno, mortalidad en el hospital y eventos adversos. No se incluyeron estudios que no fueran de naturaleza clínica (experimentales y básicos) ni estudios observacionales o retrospectivos. También se excluyeron protocolos de estudio, artículos de revisión, resúmenes, editoriales y estudios con animales.

\section{RESULTADOS}

Los resultados principales fueron fracaso del tratamiento, mortalidad en el hospital y eventos adversos. El fracaso del tratamiento se definió como necesidad de cambiar el soporte a VMI. Los datos sobre los eventos adversos incluyeron los siguientes: episodios de apnea, neumotórax, hemorragia intraventricular (todos los casos de hemorragia intraventricular y hemorragia intraventricular grave), displasia broncopulmonar (DBP) y conducto arterial persistente.

Los resultados secundarios evaluados fueron requerimiento de surfactante según evaluación clínica, duración del tratamiento no invasivo, duración de la hospitalización, duración de la estancia en la unidad de cuidados intensivos (UCI) y requerimiento de tratamiento con cafeína. 


\section{Selección de estudios y evaluación de calidad}

Dos investigadores independientes (YX y XK) revisaron y evaluaron todos los registros obtenidos. Se exploraron los títulos y resúmenes de los ensayos para excluir los estudios considerados irrelevantes y, en caso de controversia, todo el equipo buscó el consenso. Se registraron los datos de los estudios incluidos en un formulario estándar recomendado por Cochrane. ${ }^{9}$ La calidad de los ECA se revisó con la herramienta para evaluar el riesgo de sesgo de Cochrane. ${ }^{9}$ Se evaluó cada estudio para determinar (a) la generación de secuencia aleatoria (sesgo de selección); (b) el ocultamiento de asignación (sesgo de selección); (c) el enmascaramiento de participantes y personal (sesgo de rendimiento); (d) el enmascaramiento de la evaluación de los resultados relacionados (sesgo de detección); (e) los datos incompletos de resultados (sesgo de deserción); (f) la notificación selectiva (sesgo de notificación) y (g) otros sesgos.

\section{Análisis estadístico}

Las variables continuas se expresaron como diferencia media (DM) e intervalo de confianza (IC) del $95 \%$. Las variables categóricas se describieron mediante razón de probabilidades (OR) e IC95\%. Se hizo una prueba de diferencia con los resultados obtenidos para determinar la heterogeneidad entre los estudios incluidos con cada variable. Si $\mathrm{I}^{2}<50 \%$, no había una diferencia obvia. Por lo tanto, se usó un modelo de análisis de efectos fijos. Se usó un modelo de efectos aleatorios si $\mathrm{I}^{2}>50 \%$, y la prueba Q de Cochran tenía un valor $p \leq 0,1$. Dos investigadores independientes (JL y XZ) hicieron el análisis estadístico mediante una revisión sistemática de Cochrane con el programa Review Manager (RevMan; versión 5.4; The Cochrane Collaboration, 2020). Se hizo un análisis de sensibilidad para reemplazar las decisiones alternativas o los intervalos de valores de decisiones que se consideraron arbitrarios o inciertos.

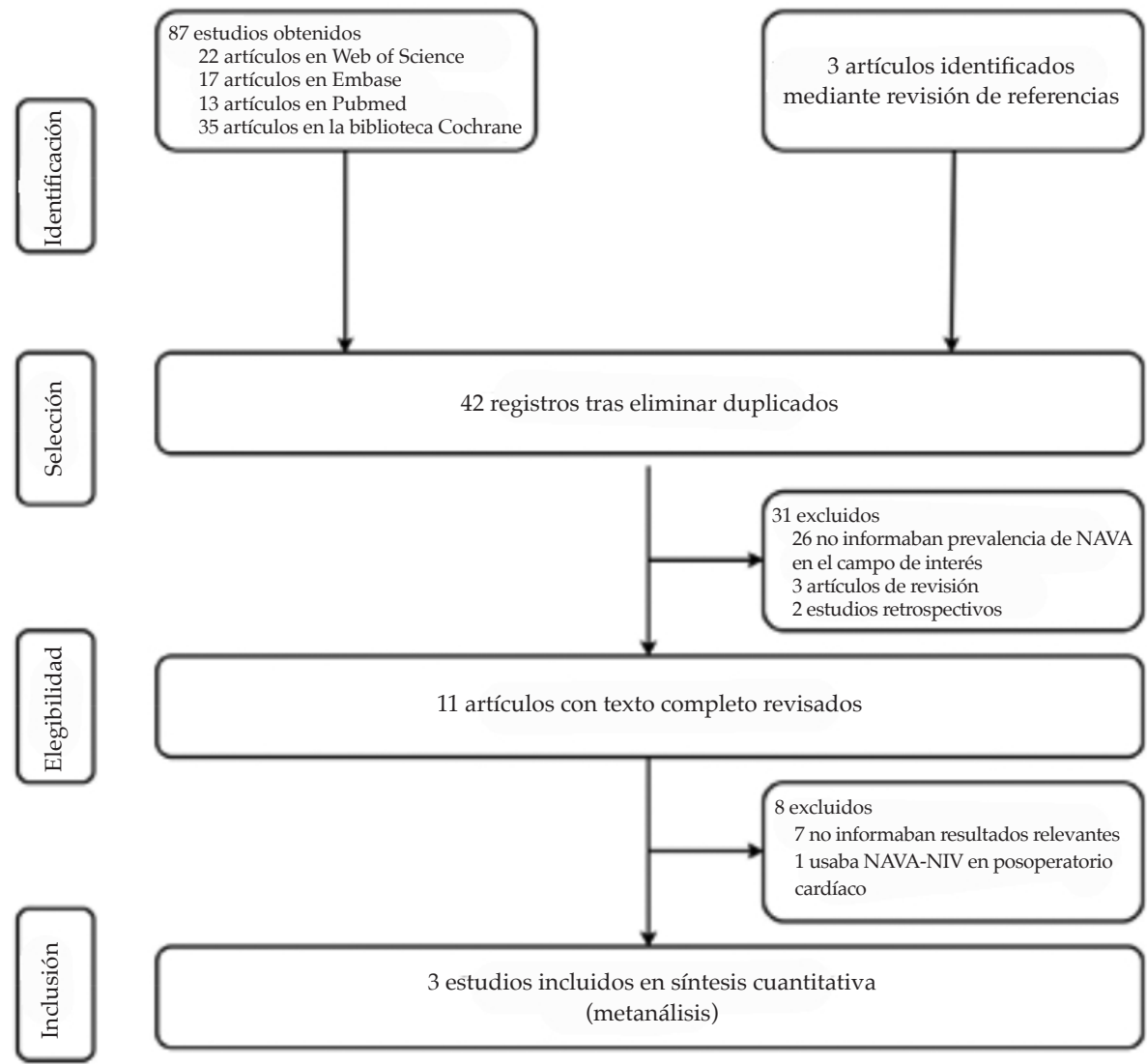

Con la búsqueda de la base de datos, se hallaron 87 artículos; con una búsqueda manual, se obtuvieron 3 artículos adicionales. Tras un proceso de cribado inicial que excluyó artículos duplicados, se revisó atentamente el texto completo de 11 artículos, que resultó en 3 artículos que cumplían los criterios de inclusión. 
92 / Arch Argent Pediatr 2022;120(2):89-98 / Artículo original

TABLA 1. Características de los estudios incluidos

\begin{tabular}{|c|c|c|c|c|}
\hline \multicolumn{2}{|l|}{ Características } & Yagui y col..$^{10}$ & Gupta y col. ${ }^{11}$ & Kallio y col. ${ }^{12}$ \\
\hline \multicolumn{2}{|c|}{ Año de publicación } & 2019 & 2019 & 2019 \\
\hline \multicolumn{2}{|c|}{ Año de experimento } & $\begin{array}{l}\text { Mayo de } 2014 \text { y octubre de } 2015 \text {, } \\
\text { octubre de } 2017 \text { y abril de } 2018\end{array}$ & $\begin{array}{l}\text { Julio de } 2014 \text { y } \\
\text { junio de } 2015\end{array}$ & $\begin{array}{l}\text { Junio de } 2012 \text { a } \\
\text { agosto de } 2015\end{array}$ \\
\hline \multicolumn{2}{|l|}{ País } & Brasil & Estados Unidos & Finlandia \\
\hline \multicolumn{2}{|l|}{ Edad } & 28-32 semanas & 26-34 semanas & 32 semanas a $36+6$ semanas \\
\hline \multicolumn{2}{|c|}{ Criterios de inclusión } & $\begin{array}{l}\text { Peso al nacer } \leq 1500 \mathrm{~g} \text {, } \\
\text { requisito de NCPAP y con } \\
\text { una fracción inspirada de } \\
\text { oxígeno } \geq 25 \% \text { en las } \\
\text { primeras } 48 \text { horas de vida }\end{array}$ & $\begin{array}{l}\text { Recién nacidos prematuros } \\
\text { que requieren ventilación } \\
\text { no invasiva, soporte en la } \\
\text { unidad de cuidados } \\
\text { intensivos neonatales }\end{array}$ & $\begin{array}{l}\text { Edad posnatal }<48 \text { h y } \\
\text { dificultad respiratoria que } \\
\text { requiere } 5-6 \mathrm{cmH}_{2} \mathrm{O}, \text { CPAP con } \\
\mathrm{FiO}_{2}>0,23 \text { para alcanzar una } \\
\mathrm{SpO}_{2} \text { de } 87 \%-93 \%\end{array}$ \\
\hline \multicolumn{2}{|c|}{ Criterios de exclusión } & $\begin{array}{l}\text { Anomalías congénitas mayores; } \\
\text { asfixia perinatal grave (puntaje } \\
\text { de Apgar a los } 5 \text { minutos }<6 \text { ); } \\
\text { padres que negaron el } \\
\text { consentimiento }\end{array}$ & $\begin{array}{l}\text { Anomalías congénitas; } \\
\text { hemorragia interventricular } \\
\text { de grado II o mayor }\end{array}$ & $\begin{array}{l}\text { Desconexión para recibir } \\
\text { oxígeno pero requerimiento } \\
\text { de CPAP; ventilación invasiva } \\
\text { antes de recibir CPAP; anomalía } \\
\text { cromosómica; anomalía } \\
\text { congénita grave }\end{array}$ \\
\hline \multicolumn{2}{|l|}{ Objetivo } & Antes de la ventilación & $\begin{array}{l}\text { Antes de la ventilación } \\
\text { (primer grupo); después de la } \\
\text { ventilación (segundo grupo) }\end{array}$ & Antes de la ventilación \\
\hline \multirow[t]{2}{*}{$\begin{array}{l}\text { Intervención } \\
\text { (NAVA-NIV) }\end{array}$} & Dispositivo & $\begin{array}{l}\text { Ventilador Servo-i } \\
\text { (Maquet, Solna, Suecia) }\end{array}$ & $\begin{array}{l}\text { Ventilador Servo-i } \\
\text { (Maquet, Solna, Suecia) }\end{array}$ & $\begin{array}{l}\text { Ventilador Servo-i } \\
\text { (Maquet, Solna, Suecia) }\end{array}$ \\
\hline & Configuración & $\begin{array}{l}\text { PPFE inicial }=5 \mathrm{cmH}_{2} \mathrm{O} \\
\text { PIM objetivo }=15 \pm 5 \mathrm{cmH}_{2} \mathrm{O}\end{array}$ & $\begin{array}{l}\text { Nivel inicial de NAVA = } \\
1 \mathrm{cmH}_{2} \mathrm{O} / \mathrm{mcV}\end{array}$ & $\begin{array}{l}\mathrm{PPFE}=5-6 \mathrm{cmH}_{2} \mathrm{O} ; \text { nivel de } \\
\text { NAVA }=0,1-2,0 \mathrm{cmH}_{2} \mathrm{O} / \mu \mathrm{V}\end{array}$ \\
\hline \multirow[t]{2}{*}{ Control (NCPAP } & Dispositivo & $\begin{array}{l}\text { Ventilador Servo-i } \\
\text { (Maquet, Solna, Suecia) }\end{array}$ & $\begin{array}{l}\text { Sistema Infant Flow SiPAP } \\
\text { (CareFusion, Yorba Linda, } \\
\text { California, Estados Unidos) }\end{array}$ & $\begin{array}{l}\text { Sistema Infant Flow SiPAP } \\
\text { (Viasys, Healthcare, } \\
\text { Pensilvania, Estados Unidos) }\end{array}$ \\
\hline & Configuración & $\mathrm{CPAP}=5-7 \mathrm{cmH}_{2} \mathrm{O}$ & $\mathrm{CPAP}=3-5 \mathrm{cmH}_{2} \mathrm{O}$ & $\mathrm{CPAP}=5-6 \mathrm{cmH}_{2} \mathrm{O}$ \\
\hline
\end{tabular}

PPC: presión positiva continua; NCPAP: presión positiva continua nasal; NAVA-NIV: ventilación asistida ajustada neuronalmente no invasiva; PPFE: presión positiva al final de la espiración; PIM: presión inspiratoria máxima.

TABLA 2. Datos basales del paciente y parámetros relevantes de la ventilación asistida en todos los estudios incluidos (NAVANIV/PPCn)

\begin{tabular}{|c|c|c|c|}
\hline Características & Yagui y col. ${ }^{10}$ & 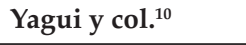 & Kallio y col. ${ }^{12}$ \\
\hline Pacientes, $\mathrm{n}$ & $59 / 64$ & 10 & $20 / 20$ \\
\hline Sexo (masculino \%) & $22(40) / 34(57)$ & $5(50)$ & $10(50) / 13(65)$ \\
\hline Peso al nacer (media $\pm \mathrm{DE}, \mathrm{g}$ ) & $1077,8 \pm 259,0 / 1130 \pm 258,4$ & $1265 \pm 403$ & $2140 \pm 766 / 2122 \pm 766$ \\
\hline $\begin{array}{l}\text { Edad gestacional } \\
\text { (media } \pm \mathrm{DE} \text {, semanas) }\end{array}$ & $29,6 \pm 2,1 / 29,8 \pm 2,1$ & $29,5 \pm 2,9$ & $33,1 \pm 2,0 / 33,0 \pm 1,8$ \\
\hline Parto por cesárea, n (\%) & $53(89) / 56(88)$ & $9(90)$ & NI \\
\hline Puntaje de Apgar al minuto, mediana (IIC) & $7(7-8) / 8(6-8)$ & $6(5-7)$ & NI \\
\hline Puntaje de Apgar a los 5 minutos, mediana (IIC) & $9(8-9) / 9(8-9)$ & $7,5(7-8)$ & NI \\
\hline Pico de AEdi (mcV) & NI & $10,8 \pm 3,3 / 15,6 \pm 7,0$ & NI \\
\hline AEdi mín (mcV) & NI & $3,1 \pm 0,5 / 3,2 \pm 1,0$ & NI \\
\hline FC (frecuencia/min) & NI & $150,6 / 146,9$ & NI \\
\hline FR (frecuencia/min) & NI & $46,2 / 49,5$ & NI \\
\hline PAM (mmHg) & NI & $44,2 / 43,7$ & NI \\
\hline $\mathrm{SpO}_{2}(\%)$ & $\begin{array}{l}95,5(94,0 ; 98,5) / 96,0 \\
(93,5 ; 97,0)\end{array}$ & $97,2 / 97,1$ & $\begin{array}{l}96,5(94,0 ; 98,0) / 96,0 \\
(93,0 ; 97,0)\end{array}$ \\
\hline $\mathrm{FiO}_{2}(\%)$ & $\begin{array}{l}24,0(21,0 ; 31,0) / 25,0 \\
(21,0 ; 30,0)\end{array}$ & $23,8 / 23,3$ & $26,0 \pm 7,0 / 26,0 \pm 4,0$ \\
\hline
\end{tabular}

PPCn: presión positiva continua nasal; NAVA-NIV: ventilación asistida ajustada neuronalmente no invasiva;

AEdi: señal de actividad eléctrica del diafragma; FC: frecuencia cardíaca; FR: frecuencia respiratoria; PAM: presión arterial media; $\mathrm{FiO}_{2}$, fracción inspirada de oxígeno; $\mathrm{SpO}_{2}$ : saturación de oxígeno; DE: desviación estándar; IIC: intervalo intercuartílico; NI: no informado. 


\section{RESULTADOS}

Se obtuvieron 87 artículos para la extracción de datos; se añadieron otros tres artículos mediante la búsqueda manual de referencias y revisiones. Se excluyeron 87 artículos por duplicación o irrelevancia según el título o el resumen. Después de analizar el texto completo de los artículos restantes, se incluyeron en el estudio tres artículos en total (Figura 1). ${ }^{10-12}$ Los estudios habían sido publicados en 2019 y representaban una amplia distribución de países. Las características de los estudios incluidos en el metanálisis se describen en la Tabla 1, y las características de los pacientes, en la Tabla 2. En conjunto, estos tres estudios que

TABLA 3. Resumen de las definiciones de fracaso del tratamiento en todos los estudios incluidos

\begin{tabular}{|c|c|c|c|}
\hline Características & Yagui y col. ${ }^{10}$ & Gupta y col. ${ }^{11}$ & Kallio y col. ${ }^{12}$ \\
\hline Oxigenación & $\begin{array}{l}\mathrm{FiO}_{2} \geq 0,40 \text { con PPC de } 7 \mathrm{cmH}_{2} \mathrm{O} \\
\text { o NAVA-NIV }\end{array}$ & $\begin{array}{l}\text { Aumento del } 10 \% \text { de la demanda } \\
\text { de oxígeno con respecto al inicio }\end{array}$ & Aumento de $\mathrm{FiO}_{2}$ a 0,4 \\
\hline Signos vitales & NI & $\begin{array}{l}\text { Frecuencia cardíaca }>80 / \mathrm{min}, \\
\text { frecuencia respiratoria }>180 / \mathrm{min}\end{array}$ & $\begin{array}{l}\text { Trabajo respiratorio } \\
\text { excesivo }\end{array}$ \\
\hline Apnea recurrente & $\begin{array}{l}\text { Dos episodios de apnea que } \\
\text { requieren ventilación con } \\
\text { presión positiva o }>3 \text { episodios de } \\
\text { apnea/h que requieren } \\
\text { estimulación táctil }\end{array}$ & $\begin{array}{l}\text { Aumento de los episodios } \\
\text { de apnea globales }\end{array}$ & Apnea frecuente \\
\hline Gasometría & $\begin{array}{l}\mathrm{pH}<7,20 \text { o } \mathrm{PCO}_{2}>65 \mathrm{mmHg} \\
\text { durante }>2 \text { horas }\end{array}$ & NI & NI \\
\hline Surfactante exógeno & Segunda dosis & NI & $\begin{array}{l}\text { Requerimiento de } \\
\text { surfactante según } \\
\text { evaluación clínica }\end{array}$ \\
\hline
\end{tabular}

NCPAP: presión positiva continua nasal; NAVA-NIV: ventilación asistida ajustada neuronalmente no invasiva; $\mathrm{FiO}_{2}$ : fracción inspirada de oxígeno.

Figura 2. Resumen del riesgo de sesgo
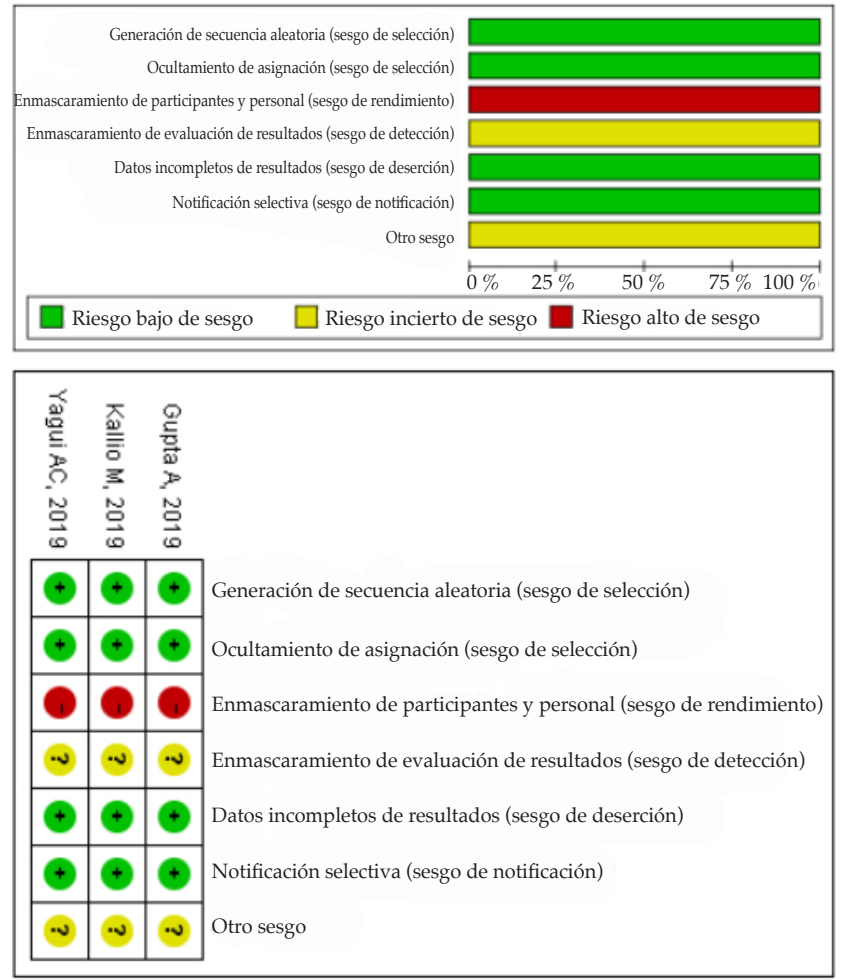

Se evaluó la calidad de los tres ensayos controlados aleatorizados incluidos en este metanálisis con el Manual Cochrane para las Revisiones Sistemáticas de las Intervenciones, versión 5.1.0. Los tres estudios presentaron un bajo riesgo de sesgo. ${ }^{18-20}$ 
cumplieron los requisitos incluían 173 pacientes (89 pacientes que recibieron NAVA-NIV y 84, NCPAP). Un estudio ${ }^{11}$ era un ensayo de grupos cruzados en el cual los pacientes estuvieron en un modo seleccionado durante un período antes de cambiar a un modo alternativo; este estudio usó la NAVA-NIV como técnica de desconexión de los recién nacidos prematuros, mientras que otros estudios usaron la NAVA-NIV antes de aplicar la ventilación mecánica.

\section{Criterios para determinar el fracaso del tratamiento}

El fracaso del tratamiento se determinó mediante signos clínicos, entre otros, necesidad de intubación endotraqueal, frecuencia respiratoria, apnea recurrente y requerimiento de surfactante exógeno. Los criterios de fracaso del tratamiento variaron levemente entre los estudios y se resumen en la Tabla 3.

\section{Calidad y heterogeneidad}

Se usó el Manual Cochrane para las Revisiones Sistemáticas de las Intervenciones (versión 5.1.0) para evaluar la calidad de los ECA. En la Figura 2 se muestran los riesgos de sesgo de los estudios incluidos. Ninguno de los estudios incluyó enmascaramiento de los participantes, los médicos ni los investigadores debido a las diferencias visibles y audibles entre los métodos de administración de oxígeno. Sin embargo, todos los estudios usaron mediciones y criterios objetivos para evaluar los resultados a modo de reducir el riesgo de sesgo de averiguación.

\section{Resultado principal}

Probabilidad de fracaso del tratamiento. Ninguno de los estudios informó diferencias en el fracaso del tratamiento (razón de riesgos $[R R]=1,09$; IC95\% = 0,65-1,84; diferencia de riesgos $[D R]=0,02$; IC95\% $=-0,10-0,14$; $\mathrm{I}^{2}=33 \%, P=0,23$; Figura 3.1).

Mortalidad. En general, la mortalidad en los estudios incluidos fue baja. No se observaron diferencias significativas en la mortalidad entre la NAVA-NIV y la NCPAP (RR $=1,52$, IC95\% $=0,51$ 4,52; no aplica heterogeneidad; Figura 3.2).

Eventos adversos. No se detectaron diferencias entre la NAVA-NIV y la NCPAP en los episodios de apnea $\left(\mathrm{RR}=1,10 ; \mathrm{IC95} \%=0,74-1,63 ; \mathrm{I}^{2}=48 \%\right.$; $P=0,17 ; 2$ estudios con 163 pacientes) (Figura 3.3). Los estudios incluidos no informaron diferencias en el neumotórax $(R R=1,38$, IC95\% $=0,33-5,83$; $\mathrm{I}^{2}=0, P=0,61 ;$ Figura 3.4). En relación con la hemorragia intracraneal, la DBP y el conducto arterial persistente, no se hallaron diferencias significativas entre la NAVA-NIV y la NCPAP (hemorragia intracraneal: $\mathrm{RR}=1,79$; IC95\% $=0$,774,18; $\mathrm{I}^{2}=0 ; P=0,73$; displasia broncopulmonar: $\mathrm{RR}=0,43 ; \mathrm{IC} 95 \%=0,09-2,15$; no aplica heterogeneidad; conducto arterial persistente: $\mathrm{RR}=0,92 ; \mathrm{IC95 \%}=0,54-1,56 ; \mathrm{I}^{2}=0 ; P=0,86$; Figuras 3.5-5.7).

\section{Resultados secundarios}

Tratamiento con surfactante. No se observaron diferencias significativas en el tratamiento con surfactante $(R R=0,88$; IC95\% = 0,58-1,36; $\left.\mathrm{I}^{2}=0 ; P=0,77\right)$. Sin embargo, se observó una tendencia de una mayor probabilidad de tratamiento con surfactante en los pacientes que recibían NCPAP en comparación con aquellos que recibieron NAVA-NIV (Figura 3.8).

Duración de tratamiento no invasivo. En un estudio ${ }^{18}$ no se informaron diferencias en la duración del tratamiento NIV entre los pacientes tratados con la NCPAP o la NAVA-NIV $(\mathrm{DM}=-20,00 ;$ IC95\% $=-76,47-36,47$; no aplica heterogeneidad, un estudio incluyó a 123 pacientes) (Figura 3.9).

Duración de hospitalización y estancia en la UCI. No se hallaron diferencias significativas entre la NAVA-NIV y la $\mathrm{NCPAP}^{20}$ en relación con la duración de la hospitalización o la estancia en la UCI (estancia en la UCI: DM $=-0,30$; IC95\% =-6,93-6,33; no aplica heterogeneidad, un estudio incluyó a 40 pacientes; hospitalización: $\mathrm{DM}=-2,00 ;$ IC95\% = -13,19-9,19, no aplica heterogeneidad, un estudio incluyó a 40 pacientes; Figuras 3.10-3.11).

Uso de cafeína. En comparación con la NCPAP, la NAVA-NIV redujo significativamente la frecuencia de uso de cafeína $(\mathrm{RR}=0,85$; IC95\% $=0,74-0,98 ; I^{2}=71 \% ; P=0,03 ;$ Figura 3.12).

\section{DISCUSIÓN}

Cada año, nacen 15000000 de bebés prematuros. ${ }^{1}$ Debido a la inmadurez del tejido respiratorio y el desarrollo de los órganos; estos recién nacidos tienen riesgo de diversas enfermedades, entre otras, SDRN, apnea y cianosis. La incidencia de SDRN aumenta con una menor edad gestacional. En un estudio de 9575 recién nacidos muy prematuros ( $\leq 28$ semanas de gestación), el $93 \%$ de los sujetos tuvieron SDRN. ${ }^{13}$ Según la Red de Investigación Neonatal, el $89 \%$ de los recién nacidos de extremadamente bajo peso al nacer 
FigURA 3. Eficacia de NAVA-NIV frente a NCPAP

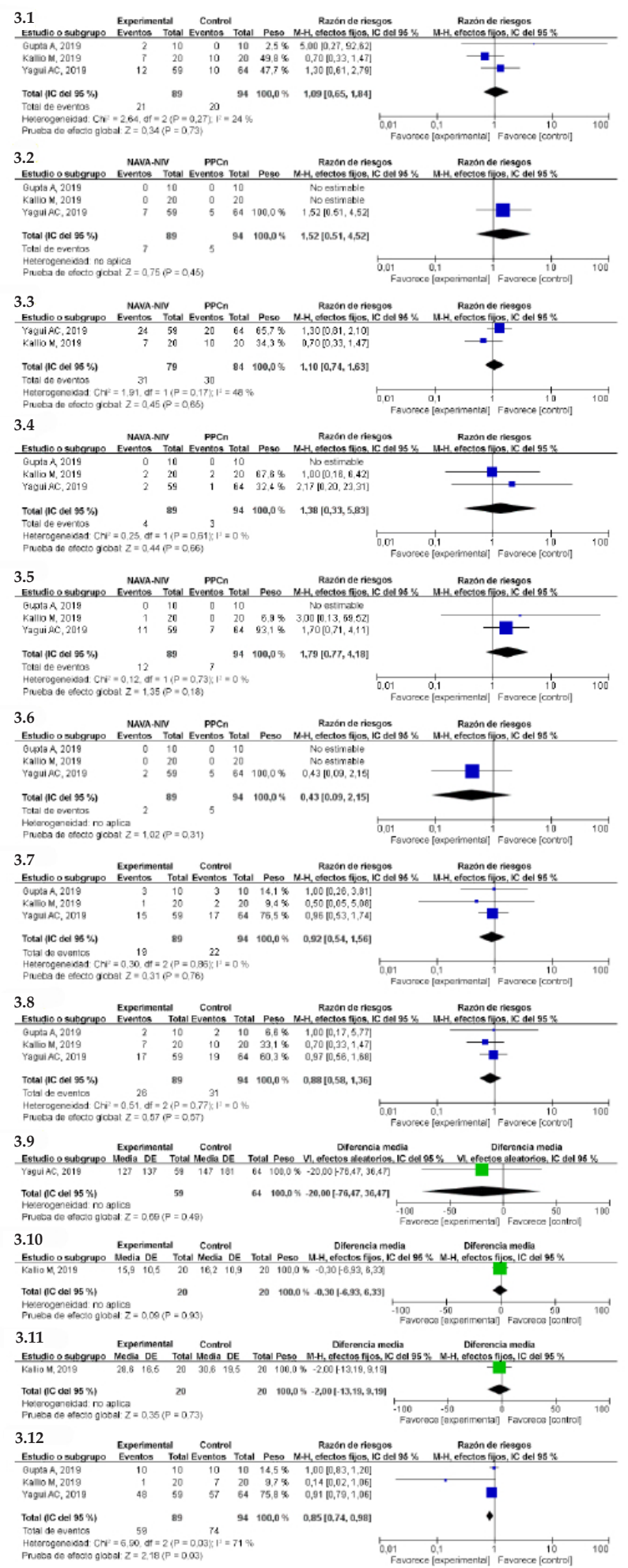

Figura 3.1 Fracaso del tratamiento; Figura 3.2 Mortalidad; Figura 3.3 Episodios de apnea; Figura 3.4 Neumotórax; Figura 3.5 Hemorragia intracraneal; Figura 3.6 Displasia broncopulmonar; Figura 3.7 Conducto arterial persistente; Figura 3.8 Tratamiento con surfactante; Figura 3.9 Duración de tratamiento no invasivo; Figura 3.10 Duración de estancia en UCI; Figura 3.11 Duración de hospitalización; Figura 3.12 Tratamiento con cafeína.

NCPAP: presión positiva continua nasal; NAVA-NIV: ventilación asistida ajustada neuronalmente no invasiva; IC: intervalo de confianza. 
recibieron VMI el primer día de vida. ${ }^{14}$ El desafío actual en el campo del soporte respiratorio de los recién nacidos prematuros es proporcionar una carga adecuada de los músculos respiratorios y un intercambio de oxígeno con sincronización adecuada del trabajo respiratorio con presión de soporte apropiado. ${ }^{15}$

Es frecuente el uso de VMI con intubación endotraqueal. Sin embargo, las complicaciones de la VMI, entre otras, lesiones pulmonares asociadas con el respirador, infecciones y DBP, pueden afectar seriamente el pronóstico a largo plazo de los recién nacidos prematuros, en especial aquellos muy prematuros. ${ }^{16-17}$

La NCPAP aumenta la capacidad pulmonar al final de la espiración, reabre las vías respiratorias pequeñas y los alvéolos colapsados y aumenta la capacidad residual funcional y la distensibilidad pulmonar; en consecuencia, mejora la ventilación y la oxigenación y reduce la derivación intrapulmonar. ${ }^{18}$ La NAVA emplea la actividad eléctrica del diafragma (AEdi) para regular la respiración. La AEdi representa la actividad neuronal y, como señal eléctrica, existe independientemente de la presión. Ambos sistemas de soporte respiratorio proporcionan ventilación mediante presión positiva para prevenir el colapso alveolar. Una característica exclusiva de la NAVA es que permite que el paciente sincronice el esfuerzo respiratorio espontáneo, y la actividad respiratoria del paciente controla el soporte inspiratorio, que influye sobre el factor de ganancia controlado por el operador (nivel de NAVA).$^{19} \mathrm{En}$ teoría, la NAVA está más cerca del modo fisiológico del soporte respiratorio. Por lo tanto, el uso de la NAVA evita la expansión excesiva de los alvéolos, reduce la respuesta inflamatoria y genera un efecto protector de los pulmones. Los experimentos con animales demostraron que la NAVA es beneficiosa para desencadenar el cambio entre la inhalación y la exhalación. El aumento de la sincronización respiratoria reduce la carga de los músculos del diafragma y podrían participar en la prevención de las lesiones pulmonares y del diafragma relacionadas con el respirador, lo que reduce la inflamación del sistema circulatorio y de los órganos distales y protege la función cardíaca y renal. ${ }^{20-21}$

En un estudio fisiológico de grupos cruzados que exploró los efectos de la NAVA en comparación con la ventilación con presión de soporte en una población pediátrica con síndrome de dificultad respiratoria aguda moderada y dificultad para la desconexión del respirador se observó que la NAVA redujo significativamente el índice de falta de sincronización, mejoró la sincronía entre el paciente y el respirador y mantuvo la estabilidad hemodinámica. ${ }^{22} \mathrm{Sin}$ embargo, debido a la inmadurez del sistema nervioso y del centro respiratorio de los recién nacidos prematuros, pueden ocurrir episodios múltiples de apnea, cuya incidencia está estrechamente relacionada con la madurez. En otro estudio, se sugirió que la NAVA no presentaba ninguna ventaja obvia en los recién nacidos prematuros y que no puede usarse en reemplazo de la NCPAP. ${ }^{23}$ En este metanálisis, no observamos ninguna diferencia significativa en el fracaso del tratamiento, la mortalidad ni los eventos adversos entre ambos grupos. Esto puede ser debido al agravamiento de la enfermedad del paciente y la necesidad de ventilación mecánica, que es inevitable. Los beneficios de la NAVA podrían limitarse a los pacientes con problemas graves de falta de sincronización y desconexión problemática, mientras que las ventajas se diluyen en otras poblaciones. ${ }^{24} \mathrm{Sin}$ embargo, los estudios analizados no se hicieron después de aplicar ventilación mecánica. Durante el proceso de iniciación del ciclo respiratorio completo, la NAVA se basa directamente en la actividad del centro respiratorio del paciente; por lo tanto, determina el volumen de humedad real obtenido por el paciente, evita la ventilación excesiva o insuficiente, reduce el cansancio de los músculos respiratorios o el soporte insuficiente de los músculos respiratorios y dificulta la desconexión. Un estudio llevado a cabo por Beck y col. demostró que la NAVA no se ve afectada por la fuga de aire y puede ser eficaz en los pacientes con fuga de aire no traumática en la unión para reducir la carga de los músculos respiratorios y coordinar la respiración entre el equipo y el paciente..$^{25}$ En una gran cantidad de ECA multicéntricos, se observó que el uso temprano de surfactante pulmonar en niños produjo una menor incidencia de neumotórax, enfisema estromal y DBP y redujo los índices de mortalidad. ${ }^{26}$

En este metanálisis, no se observaron diferencias significativas en el índice de uso de surfactante pulmonar entre los grupos de soporte respiratorio. No obstante, no se comparó la dosis de surfactante pulmonar entre los grupos. En un metanálisis reciente, se observó que la profilaxis con surfactante pulmonar en recién nacidos prematuros causó un mayor riesgo de muerte o DBP en comparación con el uso temprano de NCPAP (con el agregado de surfactante pulmonar, de ser necesario) 
$(\mathrm{RR}=1,12 ; \mathrm{IC} 95 \%=1,02-1,24 ; P<0,05) .{ }^{27}$ Como resultado, no se ha demostrado la ventaja del surfactante pulmonar profiláctico y se requieren investigaciones futuras. La comparación de la NCPAP y la NAVA-NIV llevó a una reducción significativa del uso de cafeína. Sin embargo, las pautas del tratamiento con cafeína son poco claras y están muy influenciadas a nivel subjetivo.

En este metanálisis, no observamos ninguna diferencia significativa en los índices de incidencia de eventos adversos (neumotórax, DBP, conducto arterial persistente y hemorragia intracraneal). Puede seleccionarse la presión positiva al final de la espiración (PPFE) según la señal de la AEdi; por lo tanto, en teoría, la NAVA debería presentar más ventajas en cuanto a la protección de los órganos. La PPFE elevada reduce el volumen sistólico, por lo que disminuye el gasto cardíaco. No obstante, los artículos analizados en este metanálisis no incluían discusiones profundas sobre las diferencias en la PPFE. Es necesario considerar las complicaciones de la PPFE, como el síndrome de fuga de aire pulmonar, la distensión abdominal y la lesión nasal. ${ }^{5} \mathrm{El}$ uso de opiáceos y sedantes también debe discutirse en mayor profundidad. Cabe reconocer que las complicaciones de la ventilación asistida mencionadas actualmente no constituyen una enumeración exhaustiva, y se requieren estudios futuros para determinar los parámetros de ventilación (como la PPFE) y los indicadores pronósticos a largo plazo.

Este estudio presenta ciertas limitaciones. En primer lugar, todos los análisis se basaron en una cantidad mínima de estudios cuyas cohortes y tamaño de la muestra eran relativamente pequeños. En segundo lugar, en este metanálisis, se incluyeron estudios no aleatorizados, lo que aumentó el riesgo potencial de sesgo de selección y de publicación. En tercer lugar, el tratamiento varió levemente entre los grupos. Los pacientes en grupos diferentes tienen su propia configuración del respirador. Por último, la configuración del respirador en este metanálisis dependía de la experiencia de los médicos, por lo que no se registraba en forma habitual.

\section{CONCLUSIÓN}

Debido a los datos limitados y a la evidencia de muy baja certeza, no fue posible determinar si la NAVA-NIV es un tratamiento seguro o eficaz en los recién nacidos prematuros. Se requieren ECA de envergadura y potencia adecuada para determinar si la NAVA es mejor para los recién nacidos prematuros en comparación con la
NCPAP, en especial debido a los efectos adversos potenciales y prolongados.

\section{REFERENCIAS}

1. Lawn JE, Blencowe H, Oza S, You D, et al. Every Newborn: progress, priorities, and potential beyond survival. Lancet. 2014; 384(9938):189-205.

2. Liu L, Oza S, Hogan D, Chu Y, et al. Global, regional, and national causes of under-5 mortality in 2000-15: an updated systematic analysis with implications for the Sustainable Development Goals. Lancet. 2016; 388(10063):3027-35.

3. McPherson C, Wambach JA. Prevention and treatment of respiratory distress syndrome in preterm neonates. Neonatal Netw. 2018; 37(3):169-77.

4. Committee on Fetus and Newborn; American Academy of Pediatrics. Respiratory support in preterm infants at birth. Pediatrics. 2014; 133(1):171-4.

5. Morley CJ, Davis PG, Doyle LW, Brion LP, et al. Nasal CPAP or intubation at birth for very preterm infants. $N$ Engl J Med. 2008; 358(7):700-8.

6. Terzi N, Piquilloud L, Rozé H, Mercat A, et al. Clinical review: update on neurally adjusted ventilatory assist-report of a round-table conference. Crit Care. 2012; 16(3):225.

7. Iwasaki E, Hirata K, Morikawa K, Nozaki M, et al. Postnatal physiological changes in electrical activity of the diaphragm in extremely preterm infants. Pediatr Pulmonol. 2020; 55(8):1969-73.

8. Raju TN, Higgins RD, Stark AR, Leveno KJ. Optimizing care and outcome for late-preterm (near-term) infants: a summary of the workshop sponsored by the National Institute of Child Health and Human Development. Pediatrics. 2006; 118(3):1207-14.

9. Higgins JP, Green S (eds). Cochrane Handbook for Systematic Reviews of Interventions Version 5.1.0. Oxford: The Cochrane Collaboration; 2011. Updated March 2011. [Fecha de acceso: 1 de diciembre de 2020]. Disponible en: https: / / handbook-5-1.cochrane.org/

10. Yagui AC, Meneses J, ZólioBA, BritoG, et al. Nasal continuous positive airway pressure (NCPAP) or noninvasive neurally adjusted ventilatory assist (NIV-NAVA) for preterm infants with respiratory distress after birth: a randomized controlled trial. Pediatr Pulmonol. 2019; 54(11):1704-11.

11. Gupta A, Lumba R, Bailey S, Verma S, et al. Electrical activity of the diaphragm in a small cohort of preterm infants on noninvasive neurally adjusted ventilatory assist and continuous positive airway pressure: a prospective comparative pilot study. Cureus. 2019; 11(12):e6291.

12. Kallio M, Mahlman M, Koskela U, Aikio O, etal. NIVNAVA versus nasal CPAP in premature infants: a randomized clinical trial. Neonatology. 2019; 116(4):380-4.

13. Stoll BJ, Hansen NI, Bell EF, Shankaran S, et al. Neonatal outcomes of extremely preterm infants from the NICHD Neonatal Research Network. Pediatrics. 2010; 126(3):443-56.

14. Walsh MC, Morris BH, Wrage LA, VohrBR, et al. Extremely low birthweight neonates with protracted ventilation: mortality and 18-month neurodevelopmental outcomes. J Pediatr. 2005; 146(6):798-804.

15. Tabacaru CR, Moores RR Jr, Khoury J, Rozycki H. NAVAsynchronized compared to nonsynchronized noninvasive ventilation for apnea, bradycardia, and desaturation events in VLBW infants. Pediatr Pulmonol. 2019; 54(11):1742-6.

16. Keszler M. Mechanical ventilation strategies. Semin Fetal Neonatal Med. 2017; 22(4):267-74.

17. Keszler M, Sant'Anna G. Mechanical ventilation and bronchopulmonary dysplasia. Clin Perinatol. 2015; 42(4):781-96.

18. Gleason C, Devaskar SU. Avery's Diseases of the Newborn. 
$9^{\text {th }}$ ed. Philadelphia: Saunders; 2012.

19. Barwing J, Linden N, Ambold M, Quintel M, Moerer O. Neurally adjusted ventilatory assist vs. pressure support ventilation in critically ill patients: an observational study. Acta Anaesthesiol Scand. 2011; 55(10):1261-71.

20. Beck J, Campoccia F, Allo JC, Brander L, et al. Improved synchrony and respiratory unloading by neurally adjusted ventilatory assist (NAVA) in lung-injured rabbits. Pediatr Res. 2007; 61(3):289-94.

21. BeckJ, Brander L, Slutsky AS, Reilly MC, etal. Non-invasive neurally adjusted ventilatory assist in rabbits with acute lung injury. Intensive Care Med. 2008; 34(2):316-23.

22. Spinazzola G, Costa R, De Luca D, Chidini G, et al. Pressure support ventilation(PSV) versus neurally adjusted ventilatory assist (NAVA) in difficult to wean pediatric ARDS patients: a physiologic crossover study. BMC Pediatr. 2020; 20(1):334.

23. Rossor TE, Hunt KA, Shetty S, Greenough A. Neurally adjusted ventilatory assist compared to other forms of triggered ventilation for neonatal respiratory support. Cochrane Database Syst Rev. 2017; 10(10):CD012251.

24. Demoule A, Clavel M, Rolland-Debord C, Peret S, et al. Neurally adjusted ventilatory assist as an alternative to pressure support ventilation in adults: a French multicenter randomized trial. Intensive Care Med. 2016; 42(11):1723-32.

25. Beck J, Reilly M, Grasselli G, Mirabella L, et al. Patientventilator interaction during neurally adjusted ventilator assistin low birth weight infants. Pediatr Res. 2009; 65(6):6638.

26. Sweet DG, Carnielli V, Greisen G, Hallman M, et al. European Consensus Guidelines on the Management of Respiratory Distress Syndrome-2019Update. Neonatology. 2019; 115(4):432-50.

27. BahadueFL, Soll R. Early versus delayed selective surfactant treatment for neonatal respiratory distress syndrome. Cochrane Database Syst Rev. 2012; 11(11):CD001456.

\section{Artículos seleccionados}

Los siguientes resúmenes y comentarios de trabajos seleccionados se encuentran disponibles en la versión electrónica de este número.

\section{Pediatr Pulmonol. 2021 Oct;56(10):3183-3188}

El índice predictivo de asma como instrumento subrogado de diagnóstico en preescolares: análisis de una cohorte de nacimiento longitudinal (Castro-Rodriguez JA, et al. The asthma predictive index as a surrogate diagnostic tool in preschoolers: Analysis of a longitudinal birth cohort)

Comentario: Manuela Dicembrino. Hospital de Pediatría Dr. J.P. Garrahan. Hospital General de Niños Dr. Pedro de Elizalde. Ciudad de Buenos Aires.

Pediatrics. 2021 Jul;148(1):e2021049999.

Resultados de la pesquisa de depresión y riesgo de suicidio en la atención primaria pediátrica (Kemper AR, et al. Depression and suicide-risk screening results in pediatric primary care)

Comentario: Juan Pablo Mouesca. Unidad de Violencia Familiar, Hospital de Niños P. de Elizalde. Ciudad de Buenos Aires.

JAMA Pediatr. 2021 Oct 7:e214251.

Asociación entre la pandemia por COVID-19, los índices de vacunación de rutina en la infancia y la proporción de vacunas al día, a través de 8 sistemas de salud de EE. UU. en la Base de Datos de Seguridad en Vacunas (DeSilva MB, et al. Association of the COVID-19 pandemic with routine childhood vaccination rates and proportion up to date with vaccinations across 8 us health systems in the vaccine safety datalink)

Comentario: Paula Domínguez. Comité de Docencia e Investigación, Hospital General de Niños Pedro de Elizalde. Ciudad Autónoma de Buenos Aires. 